\section{Influence of validation schemes in a laser Doppler anemometer period timing device for frequency measurement}

\author{
Prabha Venkatesh \\ Department of Mechanical Engineering, Indian Institute of \\ Science, Bangalore 560012, India
}

\section{Received 16 July 1980, in final form 6 November 1980}

\begin{abstract}
The short duration of the Doppler signal and noise content in it necessitate a validation scheme to be incorporated in the electronic processor used for frequency measurement. There are several different validation schemes that can be employed in period timing devices. A detailed study of the influence of these validation schemes on the measured frequency has been reported here. These studies were carried out by using a combination of a fast $A / D$ converter and computer. Doppler bursts obtained from an air flow were digitised and stored on magnetic discs.

Suitable computer programs were then used to simulate the performance of period timing devices with different validation schemes and the frequency of the stored bursts were evaluated. It is found that best results are obtained when the validation scheme enables frequency measurement to be made over a large number of cycles within the burst.
\end{abstract}

\section{Introduction}

Laser Doppler anemometers have been widely employed for velocity measurements in various flow situations. The Doppler signal obtained from flows with low particle concentration, such as those encountered in atmospheric measurements, naturally seeded wind tunnels etc, can be conveniently processed by digital instruments such as period timing devices or a combination of an A/D convertor (ADC) and computer (Peterson and Maurer 1975). The two basic requirements of these devices are the ability to detect a Doppler burst and to measure the frequency accurately within the burst duration.

The short duration of the Doppler signal and the noise content in it necessitate the inclusion of a validation scheme in these processors to measure the frequency accurately within the burst duration. A number of validation schemes have been suggested and incorporated in such processors. Relevant contributions include those of Brayton et al (1973), Dimotakis et al (1975), Hoesel and Rodi (1975), literature on commercially available counters (Disa counter model 55L90a and TSI signal processor counter type system 1990) and Tai et al (1977). However to evaluate, the performance of the processors with different validation schemes, it is necessary to determine the influence of the various operator controlled parameters, such as discrimination levels, measurement accuracy etc, on the measured frequency value. In an ideal processor, the operator controlled parameters should have negligible effect on the measured data. In this paper, measurements in an air flow obtained by a combination of an ADC and computer simulating the performance of period timing devices with different validation schemes have been reported. In each of these validation schemes, studies on the effect of operator controlled parameters on the measured frequency have been carried out.

\section{Validation schemes}

The period timing device used in a LDA system operating at low particle concentration employs the fact that the frequency of a signal is defined as the ratio of the number of zero crossings of the signal to the total time required for these crossings. Since the Doppler signal is emitted in bursts, it is necessary to incorporate several level detectors in a period timing device in order to make the frequency measurement within the burst duration. Further, a validation criterion which stipulates the condition for accepting a measured value has to be employed, so as to minimise the errors introduced in the measurement by the noise content in the signal. The following validation schemes, which include the condition for burst detection and validation criterion, are considered here.

\subsection{Amplitude validation scheme with level crossing detector} The performance of a counter with five amplitude levels, as shown in figure 1 , is considered here. The device is armed when the signal level exceeds the start level and the timing measurements are made on the threshold crossing. The measurement is completed at the threshold crossing following the signal peak that is less than the stop level.
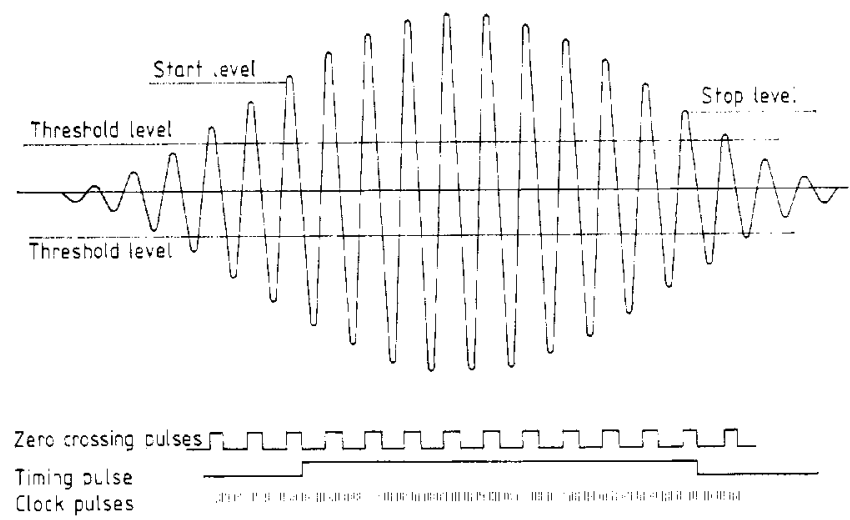

Figure 1 Amplitude validation scheme with level crossing detector.

\subsection{Amplitude validation scheme with zero crossing detector} In this scheme the period timing device has three amplitude levels - a zero level and two positive levels $A_{1}$ and $A_{2}$ (see figure 2). Level $A_{2}$ has a magnitude twice that of $A_{1}$. A zero crossing is validated only when the signal crosses the levels $A_{1}$ and $A_{2}$ in sequence with a positive gradient and is then followed by a zero crossing. The timing is commenced at the zero crossing following two valid zero crossings. Measurement is completed at the zero crossing when the input signal level falls below the level $A_{2}$ but is above level $A_{1}$. The measured data are validated only when the value is above the setting of a high pass filter which precedes the zero crossing detector.

\subsection{Time validation scheme}

Here the condition for a valid zero crossing is the same as that described in the above scheme. At the third valid zero crossing, 


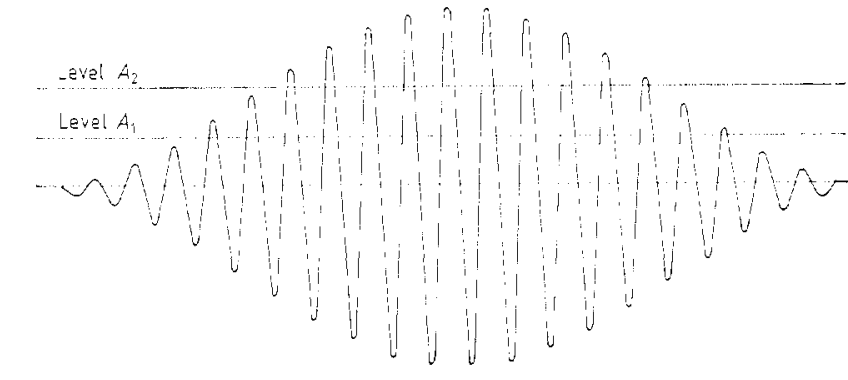

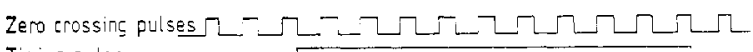

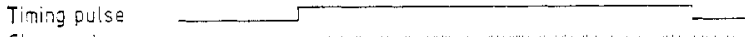

Clock f.Jisas

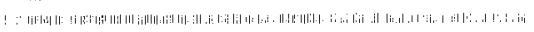

Figure 2 Amplitude validation scheme with zero crossing detector.

two counters are activated simultaneously. The first counter stops after $n_{1}$ cycles and the second counter stops after $n_{2}$ cycles. An electronic logic then compares the elapsed time $t_{n_{1}}$ for $n_{1}$ cycles to the elapsed time $t_{n_{2}}$ for $n_{2}$ cycles in the following manner.

$$
e=\frac{1}{t_{n_{2}}}\left(t_{n_{2}}-\frac{n_{2}}{n_{1}} t_{n_{1}}\right) \times 100 .
$$

This effectively expresses the percentage difference between the Doppler frequency calculated from the first $n_{1}$ cycles and the Doppler frequency from $n_{2}$ cycles. If this quantity is within $N \%$, the frequency calculated from $t_{n_{2}}$ is accepted and made available for averaging. The comparison accuracy $N \%$ is generally an operator controlled parameter with a minimum value of unity. Sometimes $n_{1}$ and $n_{2}$ are also adjustable.

\subsection{Combined validation scheme}

This scheme incorporates the desirable features of both amplitude and time validation schemes. Here the period timing device incorporates the amplitude validation scheme described in $\$ 2.2$. However, a measurement is validated only when the comparison criterion detailed in $\$ 2.3$ is also satisfied.

\section{Experimental results and discussion}

Doppler signals were obtained from air flow at a pipe outlet. The flow was sparsely seeded with silicone oil particles with diameters in the range of 0.8 to $1.2 \mu \mathrm{m}$ so that the average number of particles within the measuring control volume at any instant was one or less. The Reynolds number of the flow was 2200. A $5 \mathrm{~mW} \mathrm{He-Ne} \mathrm{laser} \mathrm{(Spectra} \mathrm{Physics} \mathrm{model} \mathrm{120)}$ was used as the source of radiation. The beam splitter and focusing lens defined a measuring control volume with a diameter of about $400 \mu \mathrm{m}$ and length of $3 \mathrm{~mm}$ at the exit of the pipe. Differential optical configuration was used and scattered radiation was focused on a photomultiplier (EMI 9683).

The main components of the electronic processing system are the Biomation 8100 transient recorder which is a fast $(100 \mathrm{MHz})$ ADC integrally connected with a digital memory and a Hewlett Packard Computer HP2116C. The contents of the recorder memory can be directly transferred to the core memory of the computer and stored on a magnetic disc with the help of suitable computer programs.

A block diagram of the processing system is shown in figure 3. The Biomation recorder was operated in "pre-trigger" mode and the trigger level was kept very low so that Doppler bursts of varying amplitudes could be recorded. It was

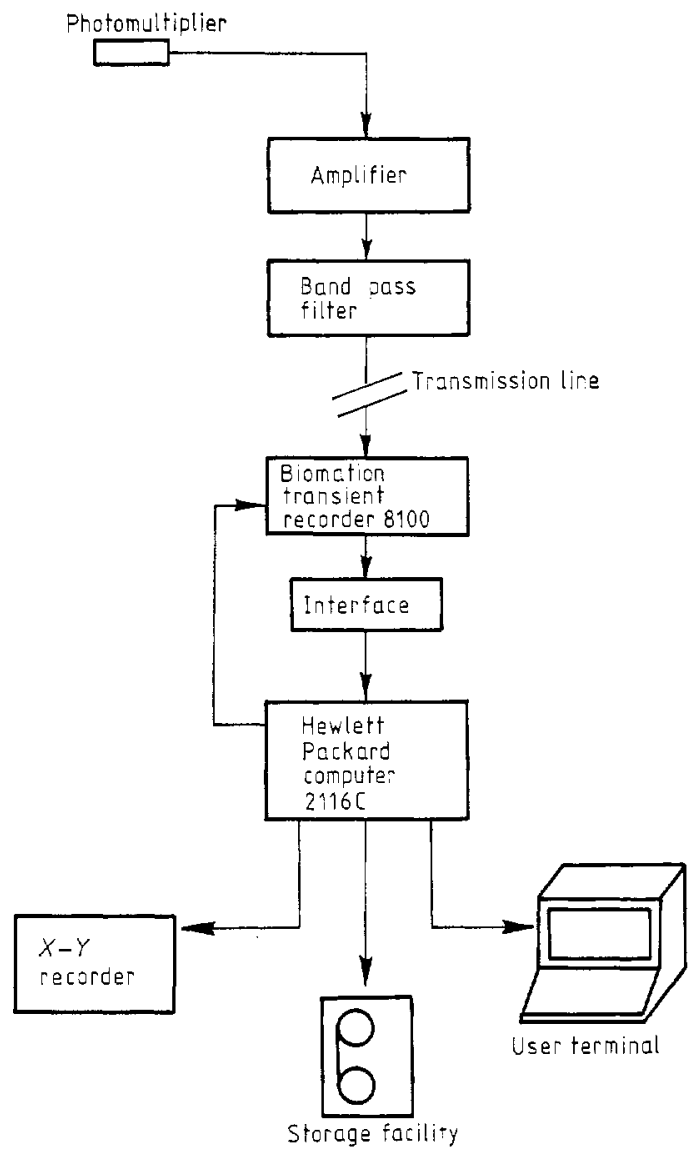

Figure 3 Block diagram of digital processing of LDA signals.

adjusted so that one Doppler burst occupied one complete memory and the sample interval enabled at least 20 digital points per Doppler cycle. The bursts were recorded at the position of the flow where the turbulence intensity was minimum. Four thousand Doppler bursts were then stored on four discs ( 1000 bursts on each disc). The operation of period timing devices with different validation schemes were simulated by suitable computer programs.

From the digitised Doppler signal, very exact zero crossings or level crossings can be calculated by fitting a straight line to a certain number of points on either side of zero. This method of period timing is therefore more accurate than the conventional counters in which the accuracy is limited to \pm 1 clock pulse. The number of points per Doppler cycle and hence the accuracy of the calculated zero crossing is determined by the sampling interval chosen while digitising the analogue signal.

The Doppler signal has a noise content which modifies the zero crossings (Cobb 1965) of the signal and can also introduce multiple zero crossings. In an attempt to overcome this problem timing measurements with respect to certain level crossings of the Doppler signal have been made (Dimotakis et al 1975, Hoesel and Rodi 1975). The use of level crossings tends to introduce small errors into the time measurement due to the changing signal amplitude within a Doppler burst and hence a changing phase position which a fixed level represents on the Doppler cycle (see figure 4). The timing error made over $n$ Doppler cycles then becomes a function of signal amplitude $A_{\mathrm{B}}$ when timing begins, the signal amplitude $A_{\mathrm{S}}$ when timing stops as well as the level amplitude $A_{\mathrm{L}}$ used to 

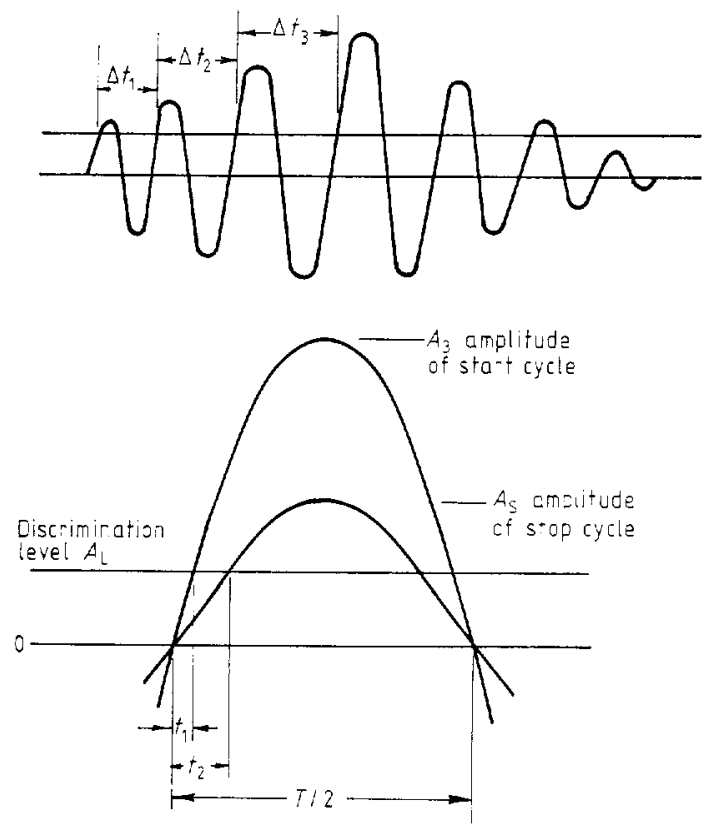

Figure 4 Period timing of Doppler signals using level crossings. Error over one period $(T), \eta=\left(t_{2}-t_{1}\right) / T=(1 / 2 \pi)$ $\left[\sin ^{-1}\left(A_{2 \mathrm{~L}} / A_{\mathrm{S}}\right)-\sin ^{-1}\left(A_{\mathrm{L}} / A_{\mathrm{B}}\right)\right]$. Error over total measurement with $n$ periods $E=\eta / n$.
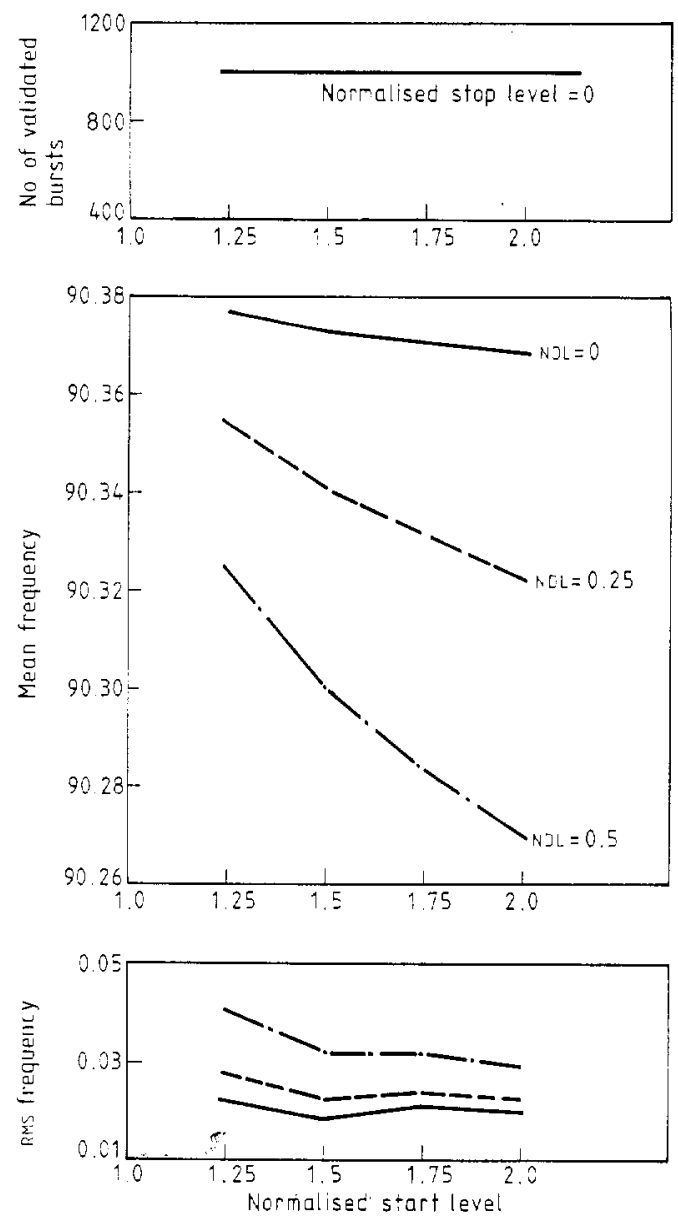

Figure 5 Performance curves for period timing device employing amplitude validation scheme with level crossing detector using simulated signals. $\mathrm{NDL}=$ normalised discrimination level.

detect each cycle. By overlapping the first and last cycle of the signal to be timed (as in figure 4), the expected timing error is calculated to be,

$$
e=\frac{1}{2 \pi n}\left[\sin ^{-1}\left(A_{\mathrm{L}} / A_{\mathrm{S}}\right)-\sin ^{-1}\left(A_{\mathrm{L}} / A_{\mathrm{B}}\right)\right] .
$$

This error is inversely proportional to the number of cycles used within the Doppler burst for timing measurement. The stop level $A_{\mathrm{S}}$ is generally less than the start level $A_{\mathrm{B}}$, so that the timing error is positive indicating that the measured frequency is less than the actual frequency. Further, for the same start and stop levels, the measured frequency decreases with increasing threshold level.

Figure 5 shows the curves obtained by simulating the performance of a period timing device incorporating amplitude validation scheme with level crossing detector. These measurements were made on electronically simulated Doppler bursts. All the amplitude levels are normalised with the Biomation trigger level. The measured mean frequency is seen to be decreasing with increasing threshold level for any particular start and stop level. In general, timing the level crossing of the signal introduces errors in the measurement due to amplitude variation of the signal within the burst and to avoid these errors, it is essential to time the zero crossings of the signal.

Figure 6 gives the experimental curves for a period timing device incorporating an amplitude validation scheme with zero crossing detector. It is observed that in $10 \%$ of the total bursts, on the average, more than one measurement per burst is made for all start levels. This can be attributed to the fact that Doppler signals always have a noise content, so that the amplitude of the signal does not increase or decrease monotonically (see figure 7). Consequently the processor can make
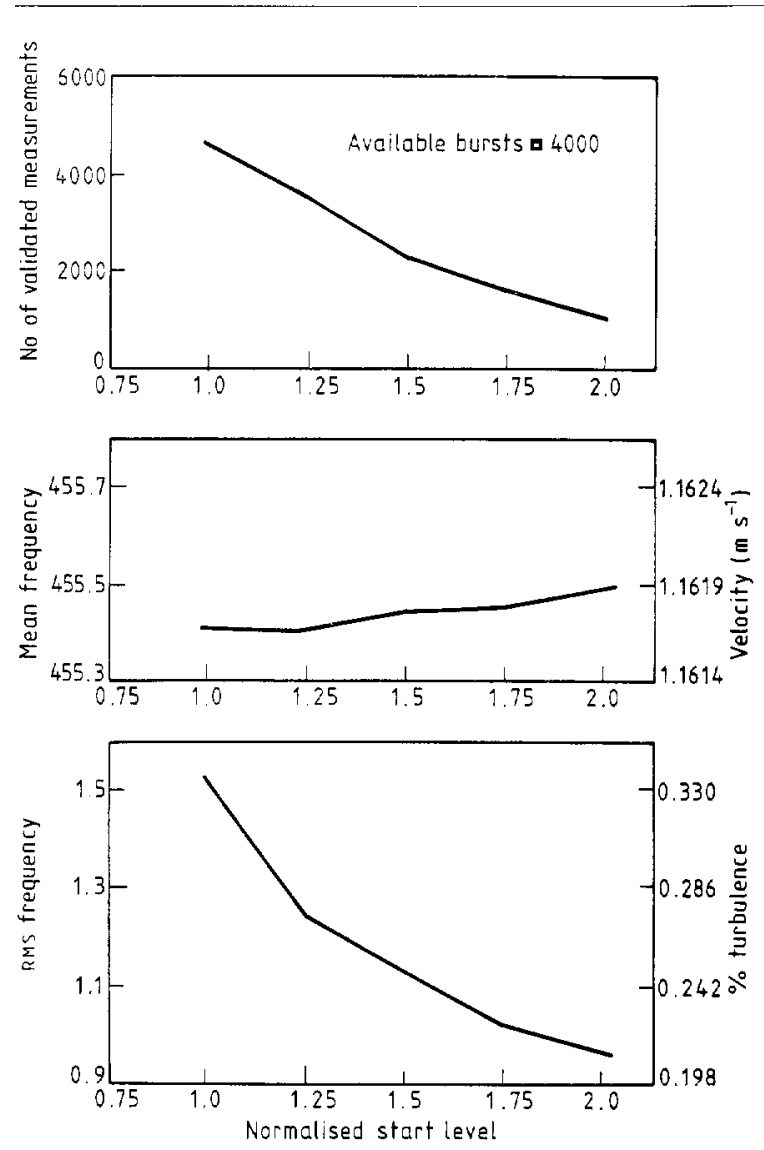

Figure 6 Performance curves for period timing device employing amplitude validation scheme with zero crossing detector. 


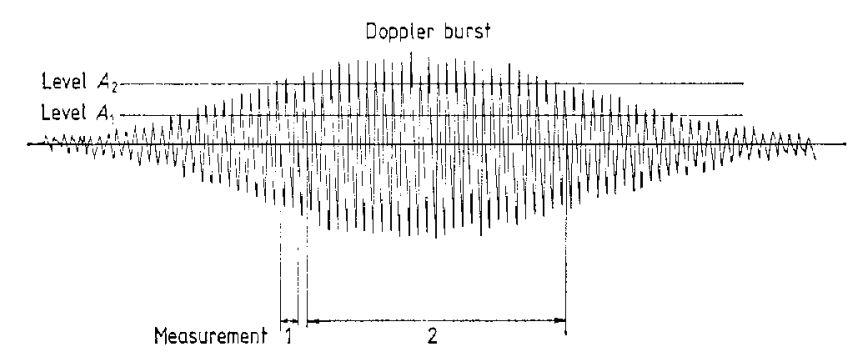

Figure 7 Possibility of multiple measurements within one Doppler burst.

a measurement over very few Doppler cycles. Since the accuracy of measurement improves with increasing number of cycles used within the burst (see Appendix) a measurement made over a few cycles in the burst can lead to significant errors in the measurement.

The computer program simulating the performance of a period timing device with time validation scheme is designed to perform 5:8, 10:16 and 15:24 comparisons using a $1 \%$ validation accuracy. Data are analysed for different amplitude levels $A_{2}$.
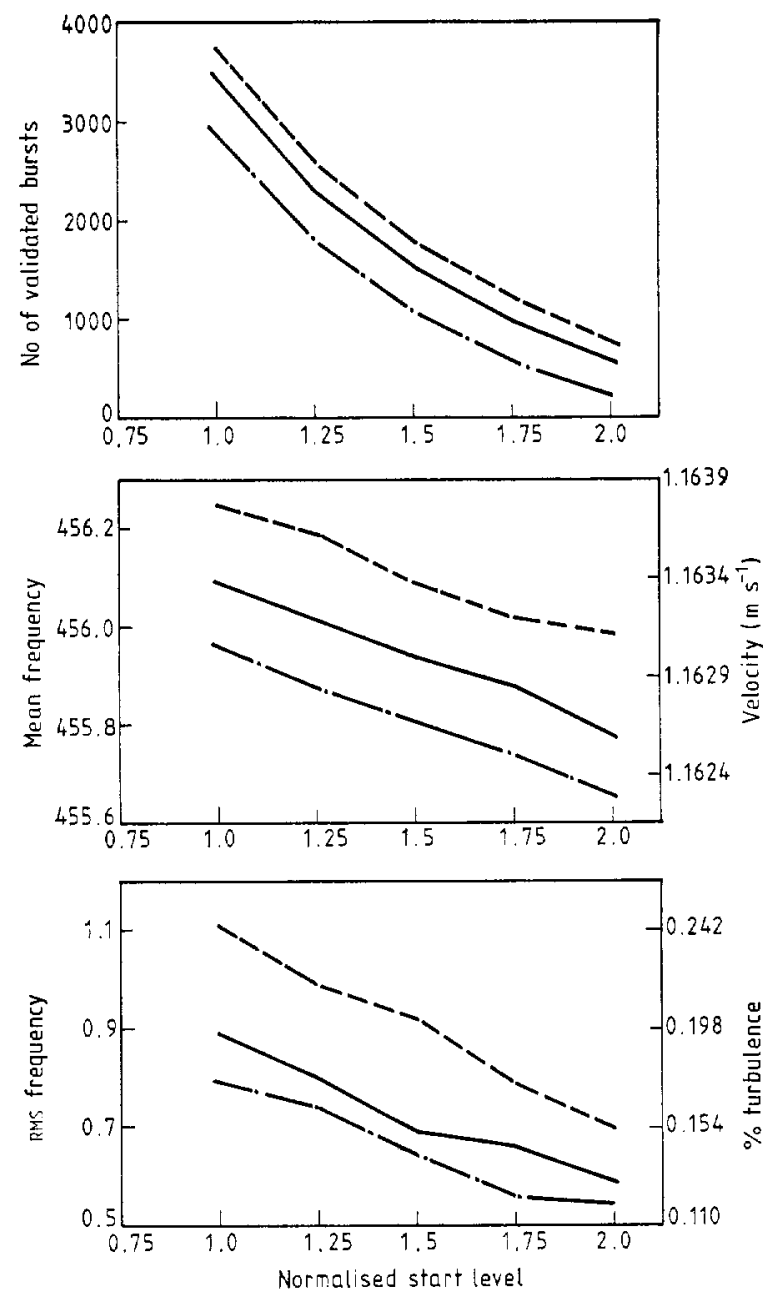

Figure 8 Performance curves for period timing device employing time validation scheme with one measurement per burst. Available bursts $=4000 .---, 5: 8$ comparison; — $10: 16$ comparison; _-. -, 15:24 comparison.
Figure 8 depicts the experimental curves when only one measurement per burst is accepted. These curves, in effect, indicate the influence of variation of signal-to-noise ratio within the burst on measured frequency. For a particular comparison mode $(5: 8,10: 16$ or $15: 24)$ measuring at higher start levels is equivalent to making measurements in the portion of the burst with higher signal-to-noise ratio. The measured mean as well as RMS frequency decreases with increasing start level and increasing number of cycles over which measurement is made.

If the period timing device resets itself within a time much shorter than the burst duration, then more than one measurement can be made within a burst. The relevant experimental curves with this scheme are shown in figure 9. Here it is
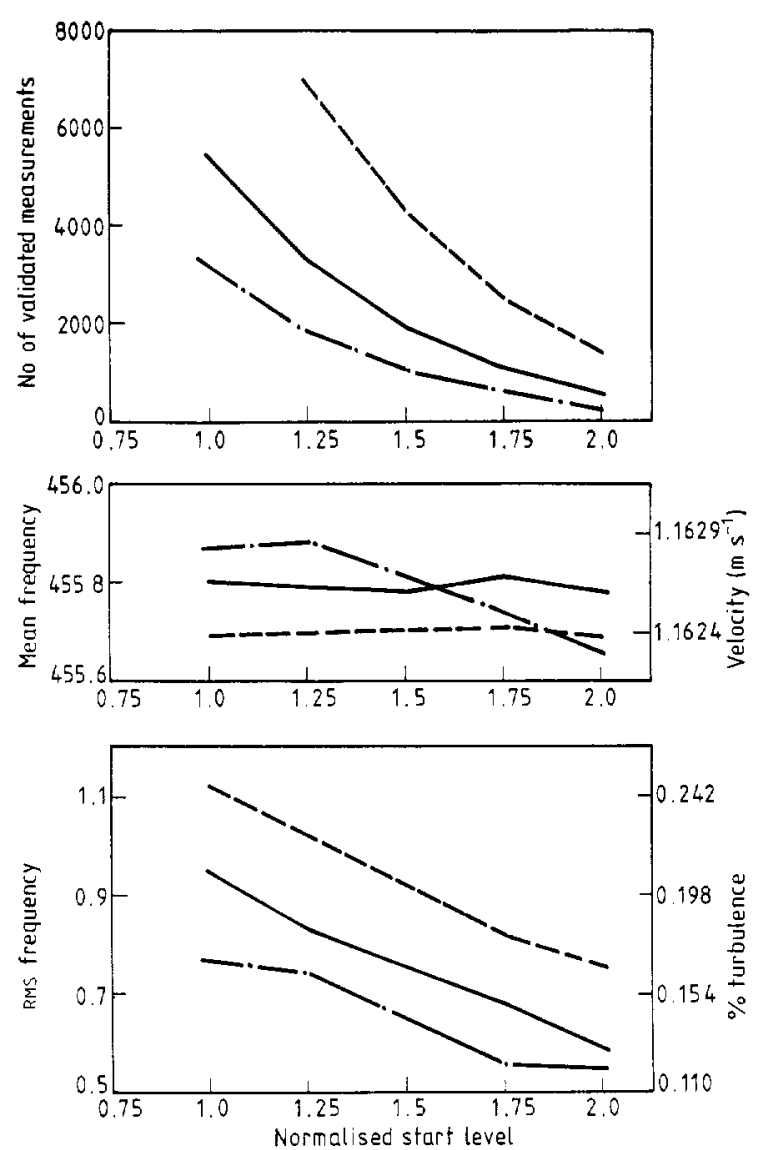

Figure 9 Performance curves for period timing device employing time validation scheme with multiple measurement per burst. Available bursts $=4000 .---$, $5: 8$ comparison; ——, 10:16 comparison; - . , 15:24 comparison.

observed that depending on the start level, the $5: 8$ comparison mode accepted between two to three measurements per burst while the 10:16 comparison mode accepted one to two measurements per burst. The 15:24 comparison mode gave only one measurement per burst and the mean and RMS frequency profile are the same as shown in figure 8 . For a particular start level and comparison mode, the number of measurements that can be made within a burst depends on the particle size. Thus the particle size distribution within the flow influences these measurements.

For the 5:8 and 10:16 comparison mode, the measured mean frequency is independent of the start level, but the the absolute value for 5:8 comparison mode is lower than that 
for the 10:16 comparison mode. The measured RMs frequency decreases with increasing start level and increasing number of cycles used within the burst. From figures 8 and 9 it is seen that the variation in the RMs frequency values with start level is not influenced by multiple measurements within the burst.

The curves obtained from computer programs simulating a period timing device with combined validation scheme are shown in figure 10 . The number of validated bursts decreases with increasing start level which is partly due to noise in the signal and partly due to validation conditions requiring a minimum of 8 or 16 cycles for measurement. For all start levels
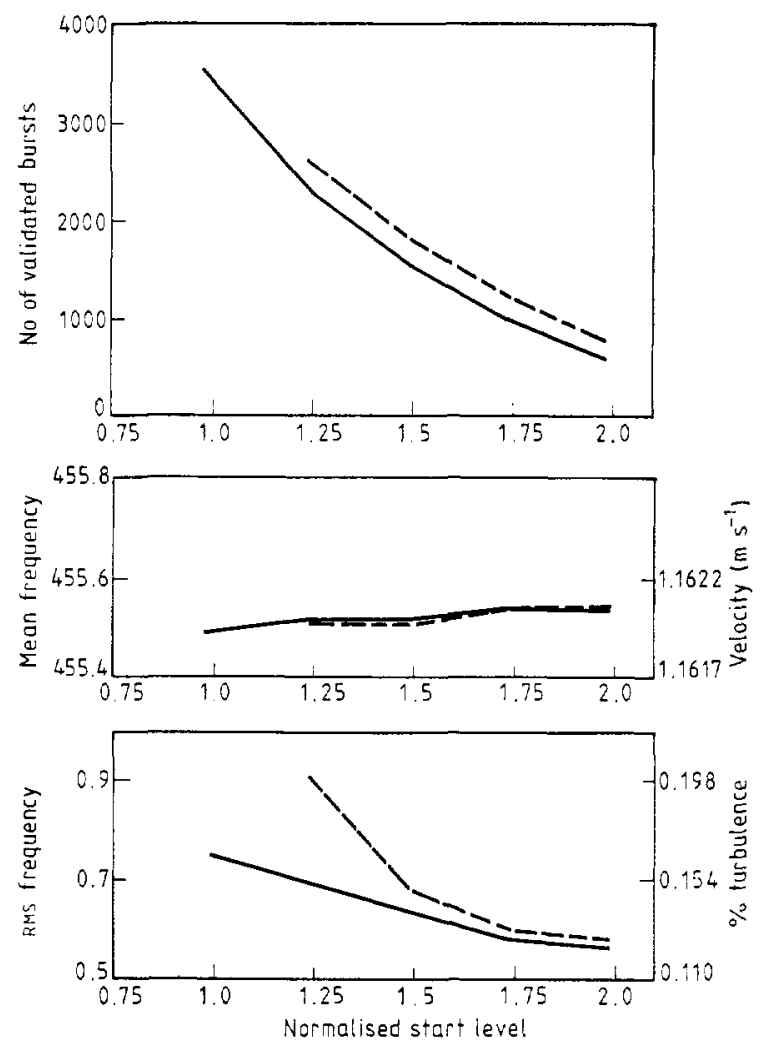

Figure 10 Performance curves for period timing device employing combined validation scheme. Available bursts $=4000 .--, 5: 8$ comparison $; \_, 10: 16$ comparison.

the number of bursts validated for the $5: 8$ comparison mode is around 200 more than validated for the $10: 16$ comparison mode indicating that these measurements are made over $n$ cycles where $8<n \leqslant 16$. Consequently the measured mean and RMS frequency with 5:8 comparison mode is higher (see figure 8) than that measured with the $10: 16$ comparison mode (see figure 9). The mean frequency increases slightly with the start level. This is due to the noise content in the Doppler burst. At low start levels, the signal-to-noise ratio is poor and it is possible that the counter makes a measurement over a few Doppler cycles (see figure 7) which is rejected as the validation criterion is not satisfied. The simulated counter continues with a second measurement at the next cycle. As the start level is increased, the portions of the burst with improved signal-to-noise ratio are utilised and the probability of making multiple measurements within the burst reduces. The measured RMS frequency decreases with increasing start level and is nearly independent of the start level at relatively high levels.

\section{Conclusion}

The following conclusions can be drawn from the results obtained with computer programs simulating the performance of period timing devices with different validation schemes.

(i) Due to"the amplitude modulation of the Doppler signal, timing the level crossing introduces errors in the time measurement and hence the measured frequency. The period timing devices should, therefore, time the zero crossings of the signal.

(ii) In the amplitude validation scheme with a zero crossing detector, due to the noise in the Doppler signal, multiple measurements are made within one burst. The measured mean frequency varies slightly with the start level. Further, the measured RMS frequency is sensitive to the start level and decreases with increasing start level.

(iii) The start level of the counter plays a significant role when only one measurement per burst is made over a few definite cycles within the burst (time validation scheme). The measured mean and RMS values decrease with increasing trigger level. Further, an increase in the number of cycles utilised within the burst for frequency evaluation is accompanied by a decrease in the measured values. The mean and RMS values measured with the $5: 8$ comparison mode is significantly higher than those measured with the 10:16 and 15:24 comparison modes. Best results are obtained when measurement is made over a large number of cycles in the portion of the burst with high signal-to-noise ratio.

(iv) In the time validation scheme, when multiple measurements per burst are made, the measured mean frequency is independent of the trigger level, but the RMS frequency is sensitive to trigger level. The RMS values measured with 5:8 comparison mode is always higher than those measured with the $10: 16$ and $15: 24$ comparison modes.

(v) In the combined validation scheme, the measured mean frequency is nearly independent of the trigger level. At low trigger levels, the RMS value measured with the $5: 8$ comparison mode is much higher than that measured with the $10: 16 \mathrm{com}-$ parison mode. However at higher trigger levels, the variation in RMS is small.

It can be surmised from the experimental results obtained here that the validation scheme incorporated in a period timing device should enable the frequency to be calculated over as many cycles in the burst as possible. Further, a measurement should be validated only if the measurement is made over a predetermined number of cycles which should be an operator controlled parameter.

\section{Acknowledgment}

The author is grateful to Professor Dr F Durst, SFB 80, University of Karlsruhe, West Germany for support and many helpful discussions during the course of this work.

\section{References}

Bendat J S and Piersol A G 1971 Random Data: Analysis and Measurement Procedures (New York: Wiley)

Brayton D B, Kalb H T and Crosswy F L 1973 Two component, dual scatter laser Doppler velocimeter with frequency burst signal readout Appl. Opt. 121145

Cobb S M 1965 The distributions of intervals between zero crossings of sine wave plus random noise and allied topics IEEE Trans. Information Theory IT-11 220

Dimotakis P E, Collins D J and Lang D B 1975 Laser Doppler velocity measurement in subsonic, transonic and supersonic turbulent boundary layers

Third International Workshop on Laser Velocimetry, Purdue University 


\section{$P$ Venkatesh}

Hoesel W and Rodi W 1975 A highly accurate method for digital processing of laser Doppler velocimeter signals Report SFB 80/E/67, University of Karlsruhe

Peterson J C and Maurer F 1975 A method for analysis of laser Doppler signals using a computer Proc. LDA Symp. Copenhagen p 312

Tai I, Hasegawa K and Sekiguchi A 1977 Digital frequency meter for burst like laser Doppler signals

J. Phys. E: Sci. Instrum. 10811

\section{Appendix}

A period timing device determines the frequency of the signal by measuring the time interval over a fixed or variable number of zero crossings. The zero crossing intervals of a signal consisting of pure sine wave and Gaussian noise have a Gaussian distribution (Cobb 1965). Thus it is possible to estimate the number $2 N$ of zero crossing intervals (samples) required to determine the sample mean $\bar{s}$ to be within a specified accuracy of the true population mean value $\mu$ in the following manner (Bendat and Piersol 1971). The confidence intervals for the estimation of the population mean are given by,

$$
\bar{s}-\frac{\sigma Z_{\mathrm{C}}}{\sqrt{2} N} \leqslant \mu \leqslant \bar{s}+\frac{\sigma Z_{\mathrm{C}}}{\sqrt{2} N}
$$

where $\sigma$ is the variance of the zero crossing interval and $Z_{\mathrm{C}}$ equals $1.645,1.96$ and 2.58 for 90,95 and $99 \%$ confidence limits respectively. Rearranging the terms in equation (A1) yields,

$$
N=\frac{Z_{\mathrm{C}}(\sigma / \mu)^{2}}{2[(\mu-\bar{s}) / \mu]^{2}} .
$$

The above equation gives the minimum number of samples required to determine with a specific confidence that the

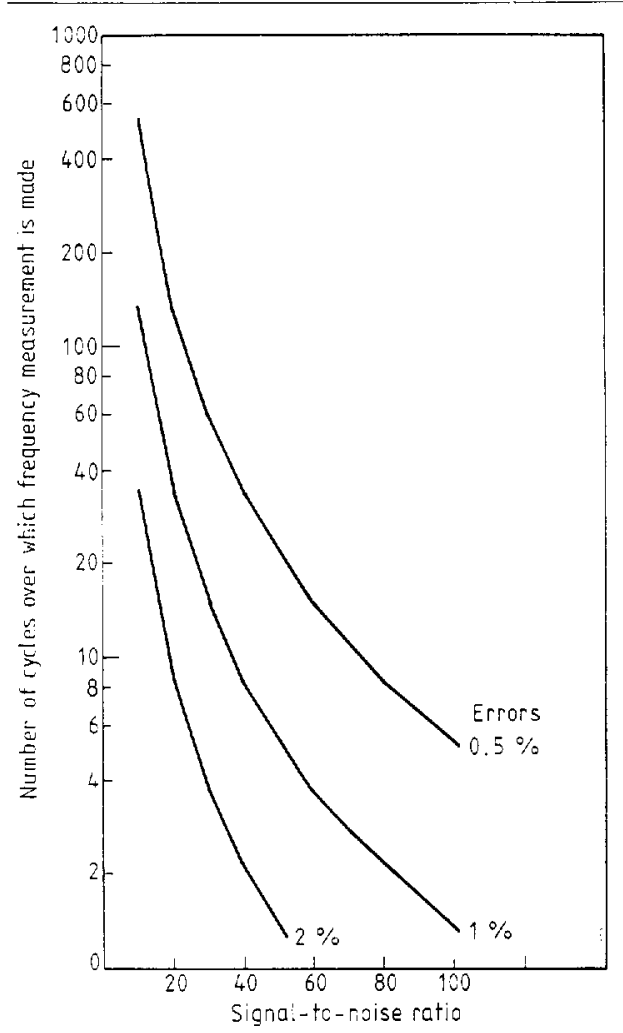

Figure A1 Effect of signal-to-noise ratio on the accuracy of frequency measurement. experimental mean value differs from the true population mean value by less than a specified percentage. Assuming the Doppler signal to be equivalent to a signal consisting of a pure sine wave with additive noise where the noise has Gaussian statistics, the following relationship is obtained (Cobb 1965).

$$
\sigma / \mu=2 / \pi a \text {. }
$$

Here $a$ denotes the signal-to-noise ratio. So that for $99 \%$ confidence level,

$$
N=\frac{(2.58)^{2} \cdot 2}{\pi^{2} a^{2}[(\mu-\bar{s}) / \mu]^{2}}
$$

Figure A1 gives a plot of $N$, the number of cycles over which measurement is made, versus the amplitude signal-tonoise ratio for $0.5,1$ and $2 \%$ errors between the measured and true frequency values.

In a Doppler burst, the signal-to-noise ratio varies within the burst and is generally maximum at the centre of the burst. If the frequency measurement is made over a fixed number of cycles within the Doppler burst, then the accuracy of measurement can be improved by making the measurement in portions of the burst where signal-to-noise ratio is high. The measurement accuracy can also be improved by measuring over a large number of cycles within the burst. 